\title{
STREAMING: VENTAJAS, DESAFÍOS Y OPORTUNIDADES DE LAS RADIOTELEVISIONES PARA CAPTAR AUDIENCIAS
}

\section{STREAMING: ADVANTAGES, CHALLENGES AND OPPORTUNITIES OF THE RADIO TELEVISIONS TO ATTRACT AUDIENCES}

Benjamín Marín Pérez. Universitat de València. España. benjamín.marin@uv.es

\section{RESUMEN}

La investigación muestra el crecimiento del consumo de streaming en los últimos años y refleja su incremento exponencial a raíz de la pandemia por la Covid 19 en 2020. La base científica cita a los autores más relevantes en esta temática y está avalada por los estudios e informes de organismos oficiales y privados de relevancia internacional. Se estudia el origen del streaming, su evolución y futuro con la ayuda de las nuevas herramientas tecnológicas, como las apps que permiten a los periodistas y al público poder entrar en directo en un informativo. Ello supone nuevas oportunidades de negocio para las radiotelevisiones generalistas creadoras de contenido en España. La investigación muestra cómo el consumo de streaming se dirige hacia el móvil, que es la herramienta para conectarse a internet que utilizan 9 de cada 10 internautas en España, siendo los vídeos cortos y los videos bajo demanda los formatos más consumidos. Es un reto para las radiotelevisiones la emisión en streaming a través de plataformas digitales multicanal que alojen contenidos para captar audiencias. Unas cadenas que tienen rasgos diferenciales respecto a las plataformas tipo Netflix o HBO, como son su carácter informativo y el menor coste de producción de contenidos por streaming.

PALABRAS CLAVE: streaming; radiotelevisiones; consumo internet; reducción costes; comunicación digital; multiplataforma digital: audiencias tv

\section{ABSTRACT}

The research shows the growth of streaming consumption in recent years and reflects its exponential increase in the wake of the Covid 19 pandemic in 2020 . The scientific basis mentions the most relevant authors on this subject and is supported by studies and reports from official and private organizations of international relevance.It also studies the origin of streaming, its evolution and future with the help of new technological tools, such as apps that allow journalists and the public to enter a live newscast. This means new business opportunities for content-creating general broadcast television stations in Spain. The research shows how the consumption of streaming is focused on the mobile phone, which is the tool used by 9 out of 10 Internet users in Spain to connect to the Internet, with short videos and videos on demand being the most consumed formats. It is a challenge for television stations to broadcast 
in streaming through multichannel digital platforms that host content to capture audiences. That channels have differential features with respect to Netflix or HBO-type platforms, such as their informative nature and the lower cost of streaming content production

KEYWORDS: streaming; broadcast radio and television companies; Internet consumption; cost reduction; digital communication; digital multiplatform; tv audiences.

\section{Cómo citar el artículo:}

Marín Pérez, B. (2021). Streaming: ventajas, desafíos y oportunidades de las radiotelevisiones para captar audiencias. Revista de Ciencias de la Comunicación e Información, 26, 45-65. http://doi.org/10.35742/rcci.2021.26.e85

\section{INTRODUCCIÓN}

La crisis del Covid-19 ha supuesto un incremento del consumo de videos online a través de streaming a nivel mundial y una proliferación de estos servicios, ya que los espectadores confinados han intensificado su transición hacia estas plataformas. En España, el consumo de streaming producido tanto por las televisiones generalistas como por las plataformas de pago de entretenimiento se disparó en un $108 \%$ a raíz del primer estado de alarma por la COVID 19 en marzo de 2020, siendo Madrid y Cataluña las comunidades donde el porcentaje es más alto (Roams, 2020). En 2020 hubo un aumento de la base de usuarios y un aumento en la oferta de las plataformas de streaming con contenido exclusivo gracias a la generación de los mayores ingresos mensuales por las suscripciones.

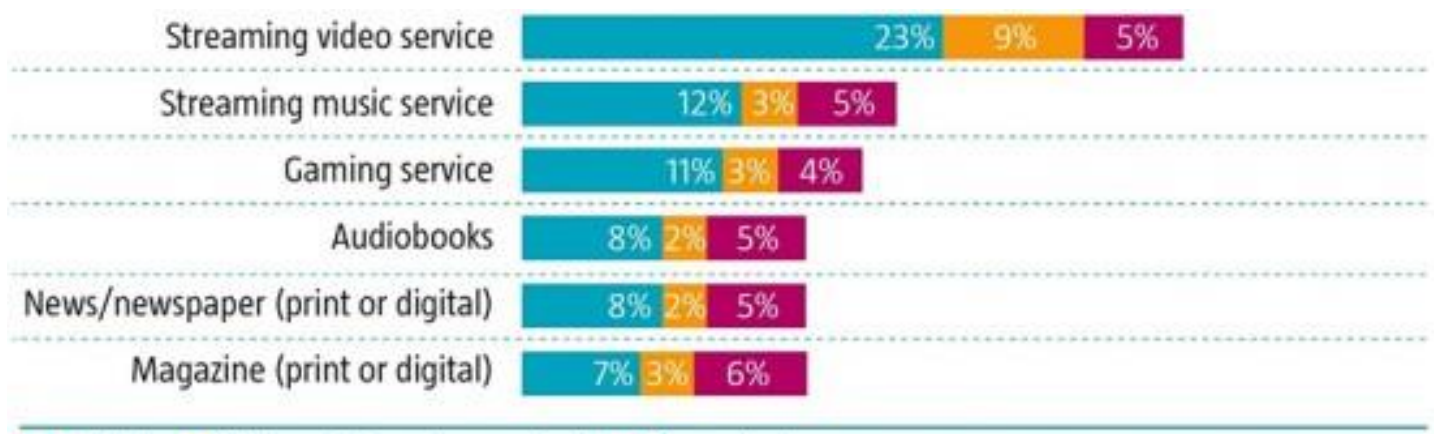

Added $\quad$ Both added and cancelled $\mathbf{\square}$ Cancelled

Gráfico 1. Variación de las suscripciones de pago desde el inicio de la pandemia de Covid-19

Fuente: Deloitte Insights, junio de 2020.

A pesar de su gran crecimiento en los últimos años se espera que el auge del streaming continúe principalmente debido al aumento de la demanda mundial de contenidos de vídeo. Según Digital TV Research (2020), los ingresos globales por series de televisión y películas online podrían duplicarse en 2025 pasando de 83 mil millones de dólares en 2019 a 167 mil millones. En 2020, el aumento fue de alrededor de 16.000 millones de dólares. 
El impacto de la crisis del coronavirus ha supuesto una revisión de las previsiones de Digital TV Research (2020) en 138 países. Según su analista principal, Simon Murray, los servicios de Vídeos Bajo Demanda (VBD) se dispararon en 2020 y aunque la confianza publicitaria se vio afectada se espera que se recupere a partir de 2021 . El estudio de Digital TV Research sobre las consecuencias de la pandemia en esos 138 países predice que cinco países controlarán dos tercios de los ingresos mundiales para 2025. El precio asequible, el amplio catálogo de contenidos, la flexibilidad de horarios, la ausencia de parones publicitarios y la comodidad de estar en tu propia casa son algunas de las ventajas del streaming respecto a la televisión de toda la vida (González, 2020).

Streaming es el nombre inglés de la trasmisión o difusión de forma continua, a través del Protocolo de Internet (IP), mediante el cual el usuario recibe el producto en su terminal a través del stream sin necesidad de descargarlo completamente. Esto se logra mediante una conexión de fragmentos de datos enviados secuencialmente a través de la red (NPLA, 2010). El streaming "real" requiere un servicio especial que difunde la información de audio/video en tiempo real. Una cadena de televisión emite en streaming, en directo, a través de su web un evento en abierto donde se puede conectar cualquier usuario. Para ello, el emisor requiere de potentes servidores con el objetivo de que el streaming funcione perfectamente y no haya cortes en la emisión.

Ahora bien, las propias cadenas de televisión disponen de un streaming denominado Protocolo de Transferencia de Hipertexto (HTTP), que es un "pseudo streaming", el cual no precisa de los potentes servidores para su utilización. Se usa a través de un protocolo TCP (utilizado para todos los ordenadores conectados a internet) para visualizar vídeos, programas, informativos, series o incluso para descargarlos. Este sistema de streaming proporciona al usuario la posibilidad de escuchar música o ver películas de forma inmediata sin necesidad de esperar a su descarga. Una característica clave para el consumo de producto audiovisual, ya que los internautas no están dispuestos a aguardar más allá de pocos segundos para ver contenido.

El factor comodidad es clave en el cambio de hábito de los consumidores. Según el productor de cine, Axel Kuschevatzky:

El consumo de los vídeos bajo demanda en el hogar ha aumentado porque la gente prefiere tocar un botón sin tener que moverse de su asiento para ir al cine... lo que ha hecho aumentar el número de plataformas en streaming. (La Nación, 2017, 5 de noviembre)

Para González (2020), estas comodidades están decantando la balanza hacia nuevos (y no tan nuevos) formatos de entretenimiento. Los usuarios pueden grabar los programas en la propia pantalla de televisión o pueden visualizarlos a través de la web de cada cadena a través del streaming cuando consideren. Ello posibilita un mayor consumo de productos, ya sea porque está en abierto o bajo el formato Pago Por Visión (PPV). En cuanto a la visualización en vivo, la masiva utilización de los terminales móviles (tabletas y teléfonos inteligentes) permite ver cualquier retransmisión siempre y cuando las cadenas de televisión dispongan de una web integrada en plataformas digitales que tengan esa posibilidad. 
Los continuos avances tecnológicos en el ámbito del streaming y la popularización de los servicios de Internet son factores que han cambiado la forma de interrelación entre las radiotelevisiones generalistas y los usuarios. Guerrero Pérez (2018) lo vincula a las cualidades disruptivas de las innovaciones tecnológicas. De hecho, los nuevos canales digitales y las redes sociales (RRSS) han contribuido al cambio de hábitos de consumo de los espectadores, especialmente en los más jóvenes (Gallardo-Camacho, Lavín, Fernández-García, 2016).

Aunque estos cambios afectan a toda la población, inciden mayormente en el público infantil y juvenil porque configuran un grupo de consumidores dentro de un mercado definido por un conjunto de características, necesidades y demandas totalmente nuevas (Gallardo-Camacho, Lavín, Fernández-García, 2016). Esa relación entre los grupos multimedia de comunicación y estos usuarios provoca impactos heterogéneos y cambios en las estrategias de las empresas.

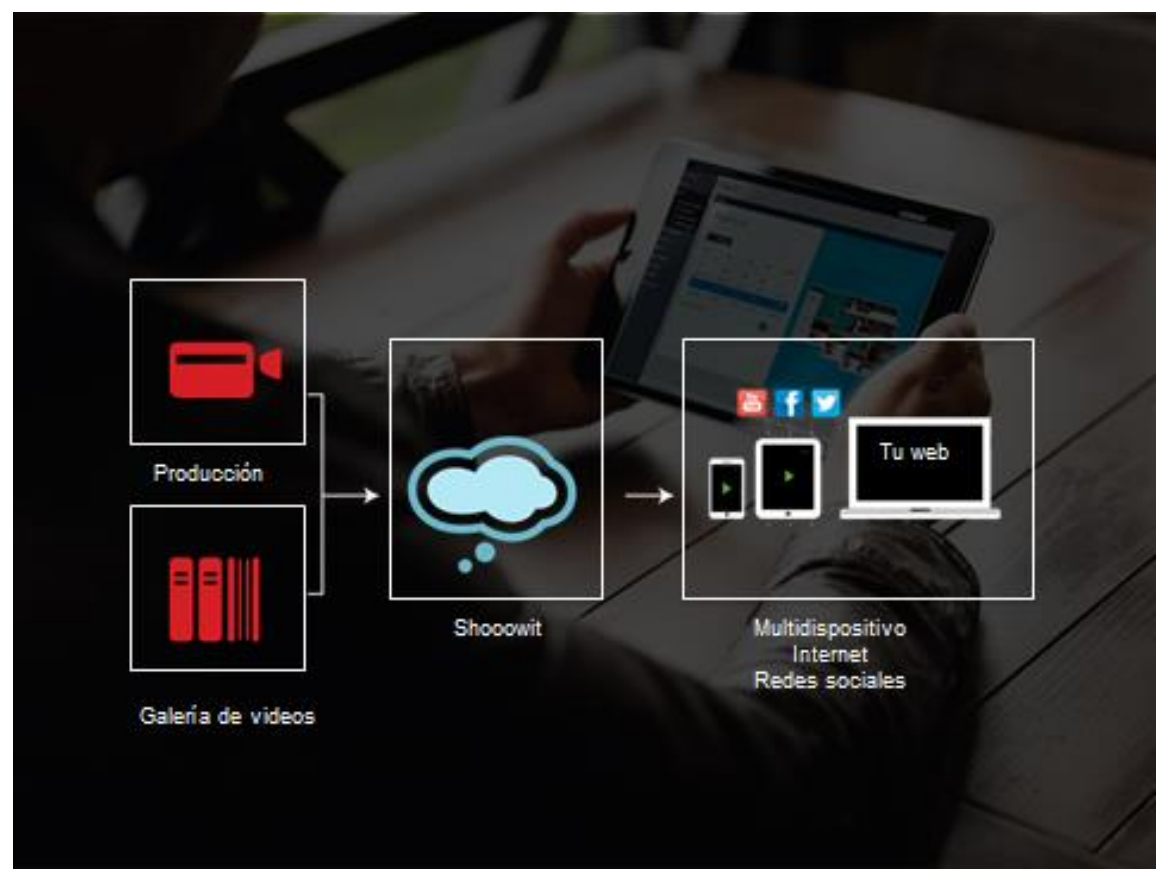

Figura 1. Proceso de creación del streaming

Fuente: elaboración propia)

Se trata de un público que ha nacido ya con la tecnología, internet, los dispositivos móviles y las RRSS. Los mayores de edad hacen transacciones, comparten productos y servicios (forman una parte integral del e-commerce). Concretamente en el target entre los 20 y los 35 años se encuentran los millennials, un público que se ha convertido en objetivo prioritario para las empresas, que instrumentan estrategias de desarrollo de contenido móvil individualizado para atraerlos y satisfacer sus necesidades y preferencias.

En ese sentido, el contenido móvil de alta calidad es un punto toral aunado a una presentación basada en estímulos visuales, interactividad, localización y las llamadas entrantes (Medina Salgado, 2016). Guerrero Pérez (2018) habla directamente de "fuga" de los millennials de la televisión lineal y Jessica Izquierdo (2017) de "abandono 
progresivo". El poder retenerlos es uno de los retos al que se enfrentan las radiotelevisiones llamadas generalistas, es decir, aquellas que se dirigen al público en general y emiten de forma lineal en abierto.

El estudio "Dimensión 2020: Media \& Me" de Kantar, realizado en los países con mayor inversión publicitaria del mundo, entre los que se incluye España, indica que el $68 \%$ de los espectadores valora la posibilidad de ver lo que quiera y cuando quiera, el $74 \%$ los utilizan para ver nuevas series o películas y un $45 \%$ los utiliza para volver a ver sus series o programas favoritos.

\section{OBJETIVOS}

El objetivo principal de la investigación es analizar el nuevo papel de las televisiones generalistas en España como creadoras de contenido para emisión en streaming con la utilización de los nuevos avances tecnológicos: las ventajas diferenciales respecto a plataformas tipo Netflix y los retos a los que se enfrentan.

Los objetivos secundarios son varios:

- Mostrar el crecimiento del consumo de vídeos por internet a nivel internacional y sus perspectivas de futuro.

- Analizar el potencial de las herramientas como la plataforma ilimitada de canales y las apps que permiten a cualquier periodista o espectador entrar en directo en una televisión.

- Analizar el nuevo rol del público que pasa a ser protagonista de la información, no solo un consumidor de contenidos.

- Mostrar el origen del streaming y su evolución hasta la actualidad

\section{METODOLOGÍA}

La investigación utiliza la revisión bibliográfica documental de autores especialistas en esta temática como Carlson, Marín Amatller, Caldwell, Creeber, Hills, Meikle, Young, Bergillos, Campos o Quintas-González, que nos ofrecen un marco teórico adecuado para abordarla. El trabajo parte del origen del streaming, cómo funciona, cómo se desarrolla a nivel internacional y cuál es la realidad de su consumo en España, ofreciendo claves de su evolución, futuro en las televisiones generalistas de nuestro país y el nuevo papel activo de los consumidores.

Como técnicas de recolección de datos, además de la revisión bibliográfica, se opta por utilizar las fuentes más representativas tanto en España como a nivel internacional en esta materia. Se trata de estudios de carácter oficial como el Informe Anual del Sector de los Contenidos Audiovisuales en España (2019), realizado por el Observatorio Nacional de Telecomunicaciones (ONTSI) del Ministerio de Asuntos Económicos y Transformación Digital. También incluye el Panel de Hogares de 2019 (último cuatrimestre) de la Comisión Nacional de los Mercados y la Competencia (CNMC). A los estudios de estos organismos oficiales se unen otros que son referencia en este campo, como los informes que realiza la Asociación para la Investigación de Medios de Comunicación (AIMC, 2020) o aquellos de prestigio internacional realizados por Digital TV Research (2020), la británica Kantar (Estudio 
Dimension 2020: Media \& Me) o la canadiense Hootsuite en unión con la británica We Are Social (Report 2020).

Por su carácter no exclusivamente teórico, se ha creído conveniente incluir entrevistas semiestructuradas a dos CEO especialistas en esta materia. Uno perteneciente a una empresa de tecnología especializada en streaming y otro relacionada con una productora que presta estos servicios a televisiones generalistas.

\subsection{Origen}

La primera patente que utilizó la palabra streaming fue en los años 20 cuando la empresa Muzak desarrolló una plataforma de música continua para negocios (conceptoweb-studio.com). La lectura en tránsito no es algo que pudiese surgir de la noche a la mañana. Antes de que este tipo de retransmisión existiese era necesaria la aparición de algunos elementos tecnológicos. En primer lugar, un ordenador capaz de reproducir el contenido.

En los años 80 ya había computadoras, pero éstas no eran lo suficientemente potentes, si bien la calidad del ancho de banda apenas permitía una conexión mínima a Internet. Las estaciones de radio por internet fueron el primer boom del streaming, ya que no se necesitaba tanta velocidad para sintonizar el audio de manera fluida. En vídeo, las primeras transmisiones en tiempo real sólo eran vistas por 2 o 3 personas, ya que no había la capacidad de hacerlo escalable (timetoast.com). Después se creó una red virtual en Internet capaz de transmitir el contenido en multicast (envío de la información en múltiples redes a múltiples destinos de forma simultánea) y los portales a los que los navegantes pudiesen acceder para disfrutar del material retransmitido (ionos.es).

Los primeros accesos a la red eran hechos a través de líneas de teléfono convencionales y era imposible hacer transmisiones en tiempo real. En 1993 se emitió, gracias a la red virtual Mbone, el directo del grupo musical Severe Tire Damage. Un año más tarde, fueron los Rolling Stones. En 1995 la empresa Real Networks transmitió por primera vez un juego de béisbol de los playoffs. Ese mismo año en Seattle se realizó la transmisión de un concierto de la sinfónica en el Paramount Theater. En 1997, RealPlayer, el primer sistema de reproducción de vídeo en streaming fue lanzado por Real Networks y también en ese período la banda Severe Tire Damage hizo historia al transmitir su concierto en vivo a todo el mundo (timetoast.com).

La lectura en continuo estaba ahí pero no se podía llevar a cabo hasta que mejorara la conexión a Internet, algo que ocurrió con la llegada del nuevo siglo. El uso de Internet se multiplicó y todo cambió. De la mano de los avances tecnológicos y el crecimiento exponencial del uso del internet y de las capacidades en hardware de las computadoras, el streaming cobró una nueva dimensión. El año 2005 fue muy importante para la proliferación de los videos gracias al lanzamiento del portal gratuito YouTube, además de la creación de los reproductores de videos basados en flash (Antolín-Prieto, 2012).

Gracias al streaming hoy existen plataformas como Netflix, Spotify, HBO, YouTube y CWS, entre otras. El contenido que se consume vía streaming es, en su práctica 
totalidad, música y vídeo, si bien, también podemos encontrar documentos de texto, pdfs, diapositivas y un largo etcétera. Todo lo que podamos crear lo podemos distribuir de esta manera, incluyendo ya partidos de fútbol, mítines, reuniones, eventos con celebridades...

\section{CÓMO FUNCIONA HOY}

A nivel técnico, la retransmisión en streaming requiere de una fuente de grabación de video y audio, un codificador, un editor y una red de distribución para entregar la retransmisión a los espectadores. Cuando la retransmisión es para miles de personas, la emisión por una radiotelevisión generalista moderna necesita de una plataforma multimedia de última generación. Hoy la tecnología permite soportes para disponer de dos canales de emisión, diez, cien o de número ilimitado (Óscar Ferrando, entrevista personal, 29 octubre 2019).

La plataforma cloud, sin instalación física de software, de gestión de vídeo por streaming en la nube permite de una forma sencilla y ágil una completa dirección desde cualquier lugar de contenido digital y audiovisual tanto en directo como a la carta. Desde la plataforma se tiene acceso a la configuración, gestión y monitorización tanto de las emisiones en directo como las realizadas bajo demanda. Una alta tecnología que permite difundir su contenido a nivel mundial sin límite de visualizaciones gracias al uso de content delivery network (CDN), que es una red de distribución de contenido formado por servidores ubicados en diversos puntos del planeta.

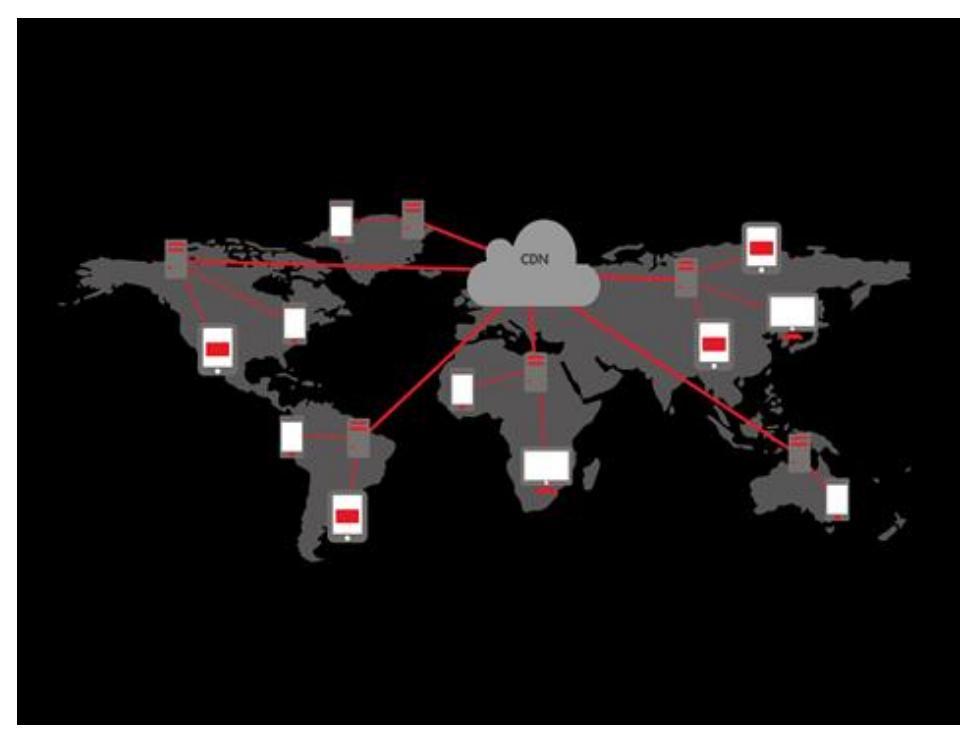

Figura 2. Red de distribución mundial CDN por servidores.

Fuente: elaboración propia

La plataforma permite la producción, codificación de señal, la distribución, la publicación y la emisión del contenido al instante sin cortes ni retrasos. Para ello, sólo necesita contar con un reproductor que sea capaz de reproducir contenido en $4 \mathrm{k}$ y $360^{\circ}$ (shooowit.com). 


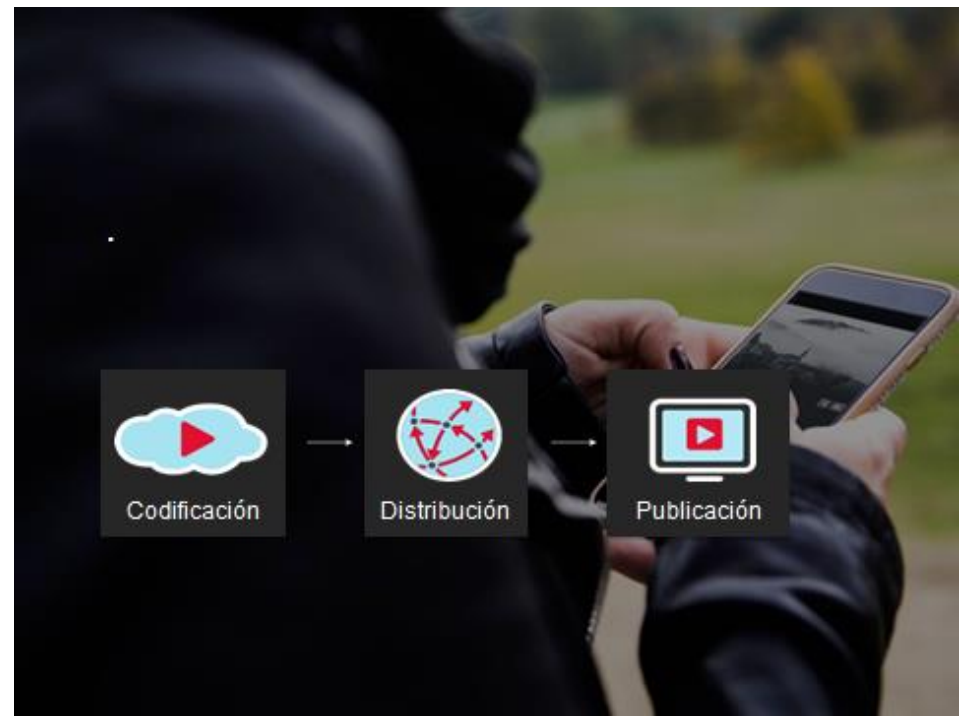

Figura 3. Las 3 fases de creación del streaming Fuente: elaboración propia

\section{EVOLUCIÓN Y FUTURO}

En la década del 2000 los usuarios tomaron el control de los contenidos de la red. El crecimiento exponencial del ancho de banda por la llegada al usuario doméstico del ADSL a baja velocidad supuso una popularización de internet y con él los contenidos multimedia y aquellos que eran emitidos en streaming. A partir de 2005 los vídeos se posicionaron mejor en las webs y aumentaron su calidad. Hasta ese momento, "el vídeo e Internet eran prácticamente dos mundos aparte, dos universos sin casi intersección" (Marín Amatller, 2005).

La llegada de la era 2.0, donde el internauta comienza a interactuar con el emisor, y se convierte en un protagonista que quiere elegir sus contenidos, supuso un punto de inflexión clave para el futuro del streaming, ya que es el comienzo de una etapa de desarrollo tecnológico con el objetivo de cubrir las necesidades que en ese momento el mercado ya precisaba. El tecnólogo Curtis R. Carlson adelantó en 2008 que Internet rompería los límites en la televisión de alta definición y que el verdadero avance se produciría cuando el vídeo pasara totalmente por internet.

Y así ha sido, porque el mayor crecimiento de tráfico de Internet presente y futuro pasa por el vídeo, y éste se consume mayoritariamente a través de las plataformas de streaming. El consumo fue creciendo a medida que internet se popularizaba. España pasó de 29 millones de usuarios en 2009 a 42,4 millones en 2020, según el estudio anual de Hootsuite \& We are Social (2020). En 2009, las películas y las series se convirtieron en el contenido digital más consumido por parte de los usuarios con un $53,6 \%$ de los españoles, el $49,4 \%$ de los cuales tenían como uso más frecuente el visionado a través de la TDT y de medios online en tiempo real (streaming). Estos datos muestran cómo el vídeo online empezaba a erigirse de forma progresiva como el producto digital estrella bajo los parámetros de la emisión en streaming. 
Miguel López aseguraba en 2010 que Google quería entrar en nuestros televisores con el objetivo de ofertar servicios online a través del monitor. En 2020 estos monitores ya reciben fuentes de todo tipo incluidas las de Internet con canales de distintas plataformas, como el propio YouTube, que emite en streaming y pertenece a Google. Llega con ello el nuevo papel que las cadenas generalistas de televisión deben asumir en connivencia con las plataformas online de contenidos. Jessica Izquierdo (2017) señala que los cambios derivados de la televisión lineal obligan a reubicarla como medio de distribución en relación con otras plataformas, especialmente internet (Caldwell, 2006; Creeber y Hills, 2007; Meikle y Young, 2008).

Una estrategia que avala el propio Informe Anual del Sector de los Contenidos Audiovisuales en España (2019), según el cual el proceso de transformación que está experimentando el mercado de la televisión incluye un conjunto de intereses compartidos para empresas de telecomunicaciones y medios de comunicación, ante la creciente influencia del uso de las tecnologías IP. Tecnologías que incrementan el papel del streaming y la orientación al cliente. Según este informe de 2019, 1.655 millones de hogares en todo el mundo disponían de TV digital en el mundo en 2018, suponiendo el $88,9 \%$ de todos los hogares que cuentan con televisor mientras que la previsión es que en 2022 sean 1731 millones.

Dentro de estos servicios emerge el VBD, que impulsa el crecimiento, ya que crece a ritmos muy superiores a la televisión lineal. Si bien, se espera que la competencia de los over the top (OTT), que se refiere a los servicios y contenidos que se transmiten en banda ancha sin que las operadoras puedan controlar su distribución), obstaculice considerablemente el impulso del mercado general de televisión debido a la creciente popularidad de este tipo de servicios. De la fusión de la televisión e Internet surgen nuevos modelos televisivos online e interactivos que permite un consumo personalizado a través de una amplia variedad de pantallas (Diego, Guerrero y Etayo, 2014).

El vídeo móvil (películas, documentales, anuncios, videoclips...) llega a través de las plataformas de streaming o las propias radiotelevisiones como creadoras de contenido. Unas plataformas que crecen y obligan a las propias cadenas a idear estrategias para potenciar nuevos servicios. Según los datos del estudio "Dimension 2020: Media \& Me" (Kantar) las cadenas de TV generalistas en España siguen dominando el panorama audiovisual de TV y vídeo, las ofertas que se adaptan a los gustos individuales no paran de crecer. No faltan contenidos de TV y vídeo; de hecho, aumentan cada día. Como respuesta a este desafío, las cadenas de TV están tomando impulso.

En 2020, cuatro de las mayores cadenas de TV del mundo han lanzado nuevos servicios de streaming de suscripción (SVOD) con contenidos exclusivos. EI VBD ya no constituye un segmento pequeño en el gran ecosistema de contenidos de TV y vídeo. Los servicios de streaming y suscripción están totalmente normalizados y la competencia aumenta, ya sea en forma de entretenimiento para todos los públicos (con Netflix a la cabeza) o de servicios más específicos para todos los gustos.

El Panel de Hogares elaborado por la Comisión Nacional de los Mercados y la Competencia (CNMC), con datos del cuarto trimestre de 2019, ha analizado el 
consumo audiovisual de los españoles a través de plataformas online de pago, como Netflix, HBO, Movistar o Amazon Prime Video. A finales de 2019, este tipo de plataformas de pago estaban presentes en el $40 \%$ de los hogares con acceso a Internet. El crecimiento interanual fue de casi un $28 \%$. De hecho, si comparamos estos datos con los de 2016, ese incremento ha sido del $237 \%$

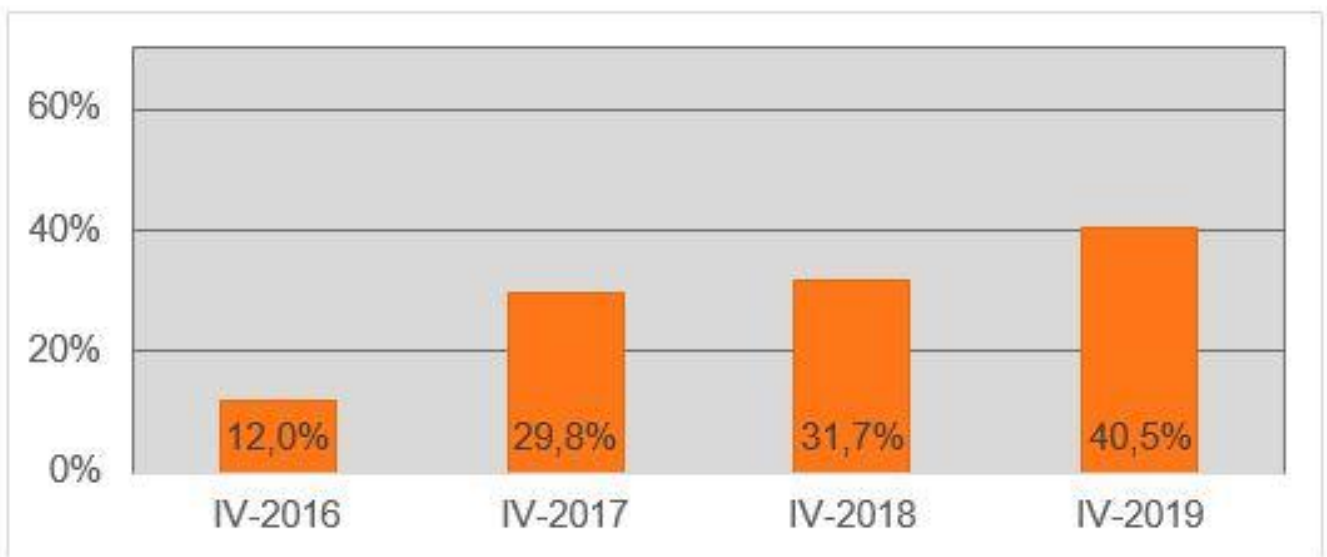

Gráfico 2. Uso de plataformas de pago para ver contenidos audiovisuales online (\% de hogares). Universo: Hogares con acceso a Internet

\section{Fuente: CNMV}

Esta encuesta publicada por el CNMV el 20 de mayo de 2020 refleja la situación de mercado antes del estado de alarma provocado por la pandemia de COVID-19. La obligación de guardar cuarentena en casa provocó un mayor uso de plataformas de streaming y disparó el consumo televisivo. Cada vez hay más individuos que consumen contenidos audiovisuales a través de Internet (en general, ya sea de manera abierta o de pago). Casi la mitad de españoles $(48,3 \%)$ consumieron contenidos online al menos una vez a la semana. En este sentido los videos cortos son los contenidos más habituales (55\%).

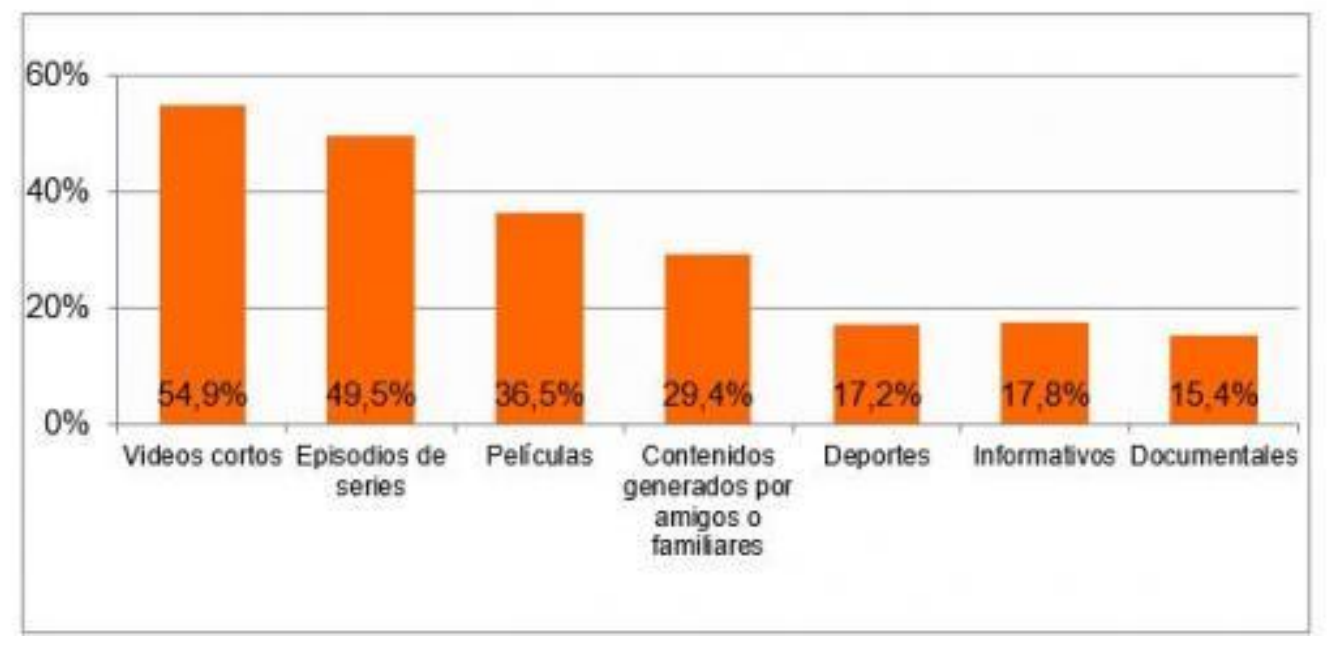

Gráfico 3. Contenidos audiovisuales online más consumidos (\% de individuos, IV2019). Posible respuesta múltiple.

Fuente: CNMV 
Los resultados del Panel también confirman que los españoles están enganchados al móvil: 9 de cada 10 internautas suelen conectarse a Internet a través de su teléfono móvil de manera habitual.

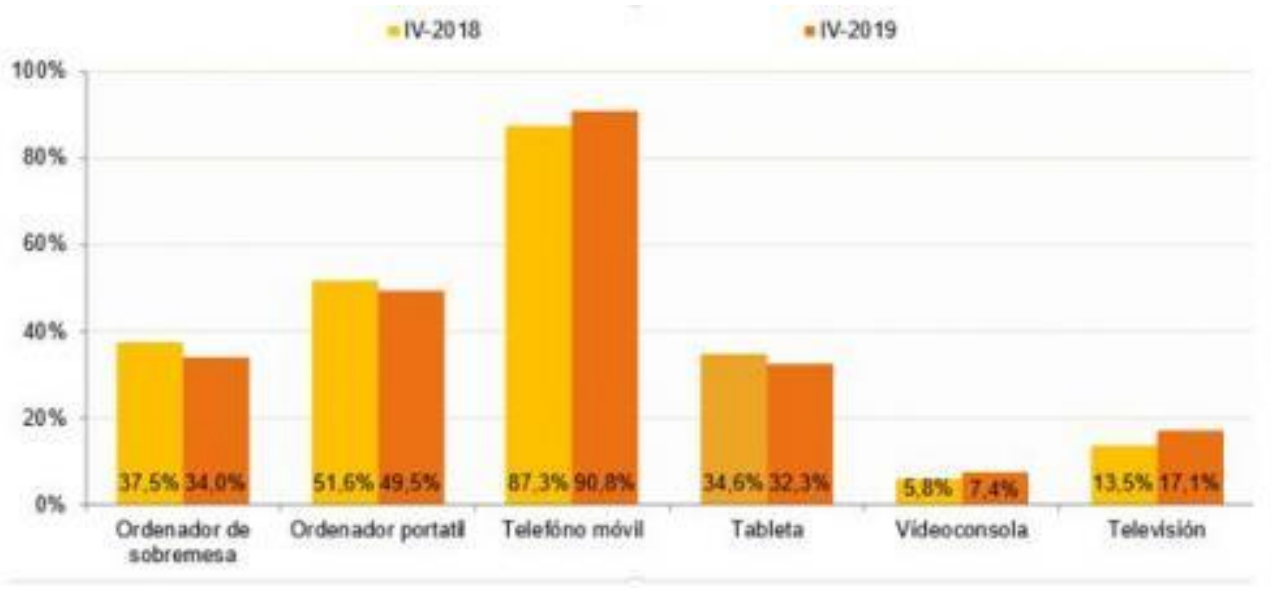

Gráfico 4. Dispositivos con los que el usuario se suele conectar a Internet (\% de individuos). Universo: Individuos que usan Internet al menos semanalmente. Posible respuesta múltiple.

Fuente: CNMV

Ante estos datos, las radiotelevisiones como productoras de contenido tienen elementos diferenciales respecto a las plataformas de contenido estrictamente por streaming y tienen a su disposición esta misma tecnología para captar nuevas audiencias o retener a las que ya tiene. Las cadenas de tv utilizan portales digitales para dar cabida a toda la oferta a través de la web (vídeo, audio, post, fotos) enlazados con las RRSS para conseguir interacción con sus públicos externos. "La participación de la audiencia ya no es anecdótica en la producción de contenidos televisivos, sino que cada vez se tiene más en cuenta" (Bergillos, 2015, p.97). En el ecosistema digital y en red, los medios de comunicación y en concreto las televisiones, buscan un modelo aún no definido donde la interacción con sus públicos es muy relevante.

Las cadenas de televisión disponen de potentes departamentos de RRSS, donde cada vez más impera el vídeo, que les ayudan en su estrategia de difusión de contenidos. Estos grupos multimedia de radiotelevisión disponen de plataformas digitales avanzadas tecnológicamente. Algunas de ellas permiten la trasmisión en vivo o VBD aunque ninguna televisión en España contiene canales ilimitados de emisión para llegar a todos sus targets, circunstancia que haría aumentar más el tráfico digital hacia su web, aunque sí existe esa tecnología para poder tenerlos (Óscar Ferrando, entrevista personal, 29 octubre 2019).

\section{VENTAJAS Y OPORTUNIDADES}

Según se ha visto hay autores que inciden en que la televisión lineal generalista ha de asumir su nuevo papel como medio de distribución en relación con otras plataformas pero no se debe obviar que la tv tiene también márgenes de crecimiento para la captación de audiencias unido a dos factores: su potencialidad para la creación de 
contenidos propios con el uso de la tecnología streaming y su carácter informativo como mass media que la diferencia de las plataformas dedicadas básicamente a ofertar productos de entretenimiento.

La televisión continúa siendo un medio de gran consumo y permanece como primera opción de ocio con una penetración del 85,4\% entre los españoles (AIMC,2020), lo que le confiere un papel protagonista en la sociedad y constituye un medio central para la información y el entretenimiento (Jessica Izquierdo, 2017). La televisión abierta lineal "sigue siendo un sistema vertebral de la industria audiovisual y de la comunicación e información de interés general en el ámbito de la Unión Europea" (Campos, 2017, p.148).

\subsection{Canales ilimitados}

Las televisiones disponen de plataformas de última generación donde alojar todos los contenidos generados tanto en directo como en diferido y colgados en la web. Sirven como oferta para la ciudadanía, ofreciendo una amplia variedad de temas, ya sea de todo tipo de programas o informativos sobre lo que ocurre en cualquier parte del mundo (fiestas, eventos, presentaciones, sucesos, celebraciones, retransmisiones). Para emisiones en directo, las plataformas tecnológicas permiten a las televisiones generalistas hoy la creación de diferentes canales live (en vivo) de forma ilimitada y al instante, así como en diferentes calidades para que se adapte al tipo de conexión a internet de cada usuario.

En cuanto al VBD, el usuario tiene la opción de grabar, editar y compartir en RRSS las emisiones en directo desde la nube mientras que el flujo de trabajo puede ser gestionado desde un único panel central. Esa opción del usuario le otorga un protagonismo de interacción con el medio de comunicación que consume. El sujeto de la información no es un ser pasivo que se limita a ver la televisión, sino activo porque participa y además genera información (Quintas, González, 2014, p.84). La comunicación $360^{\circ}$ de una cadena con este tipo de tecnología la convierte en una potente herramienta de difusión y promoción de contenidos. La programación de los canales en directo puede a su vez emitirse por las propias redes sociales cuando así lo estimen por la importancia del evento con el engagement que ello produce con sus consumidores.

La segmentación de contenidos a nivel tecnológico ofrece tantas posibilidades, que una televisión puede tener en su web p. ej. una sección de Deportes con 5 canales, una de Política donde se emitan en directo a la vez 4 señales distintas, una sección de Eventos Especiales, y otra de Cultura. Por ahondar en el ejemplo, esta última sección mencionada puede tener a su vez 15 canales distintos destinados cada uno de ellos a las siguientes temáticas: cine de acción, música clásica, ópera, cine español, series juveniles, teatro clásico, teatro contemporáneo, teatro infantil, artes plásticas, mimo, dibujos animados, marionetas, circo, hip hop infantil y danza clásica juvenil. Tecnológicamente es factible, otra cuestión es que sea capaz o pueda ofertar tal cantidad de productos.

Ése es otro de los grandes desafíos que tienen las radiotelevisiones generalistas para poder competir como productoras de contenido en streaming ante el avance de las 
plataformas por internet tipo Netflix o HBO. El contenido de cada uno de esos canales que comentamos de emisión por streaming puede llegar por la producción propia de cada tv, de forma gratuita por convenios de colaboración p. ej. con el Teatro del Liceo de Barcelona, el Palau de les Arts de València o el Teatro Real de Madrid a través de los cuales se pincha su señal, o por la compra de derechos de emisión de espectáculos en directo o grabado con origen en la Ópera de París o el Musikverein de Viena para su concierto de año nuevo, p. ej.

Tal oferta de canales y productos concretos, unido a una adecuada estrategia de marketing digital ad hoc, contribuiría a generar tráfico hacia la web y con ello retroalimentar la radio, los medios digitales y la televisión, generando nuevas audiencias e ingresos, ya sean desde España o desde cualquier lugar del planeta porque internet no tiene fronteras y la emisión de sus productos puede ser en cualquier idioma. Además, a través de la web se puede acceder a los programas, informativos y retransmisiones ya emitidas, y a todo el material histórico digitalizado que cada televisión considere que debe estar ahí para ser consumido de forma gratuita o de pago por visión. La oferta es enorme: visualización de todos los canales en directo y a través de las RRSS con posibilidad de contenido online, a La Carta o compra individualizada de servicios de PPV, así como productos exclusivos de cada cadena dentro de su política de e-commerce.

\subsection{La democratización del streaming}

Las nuevas tecnologías de streaming permiten a las televisiones una mayor proximidad de la información al ciudadano, mayor inmediatez, carácter internacional y un protagonismo más grande de la ciudadanía.

- Proximidad: la alta tecnología permite conseguir que los ciudadanos estén informados con sus smartphones y tablets en tiempo real a través de la web, potenciando una relación de proximidad con los usuarios a través de la retransmisión online multicanal que incluye todas las RRSS.

- Información inmediata: los canales ilimitados permiten la retransmisión en directo por streaming de noticias, eventos e información a través de los medios digitales, RRSS, radio y televisión.

- Protagonismo de la ciudadanía: los ciudadanos pueden ser los protagonistas en la tv, ya que una app descargable por cualquier usuario puede convertirlos en periodistas: "Cuéntanos con tu móvil qué está pasando". Desde cualquier lugar del mundo, a través de un simple teléfono móvil, los telespectadores pueden entrar en directo a través de esta herramienta para contar qué está sucediendo. 


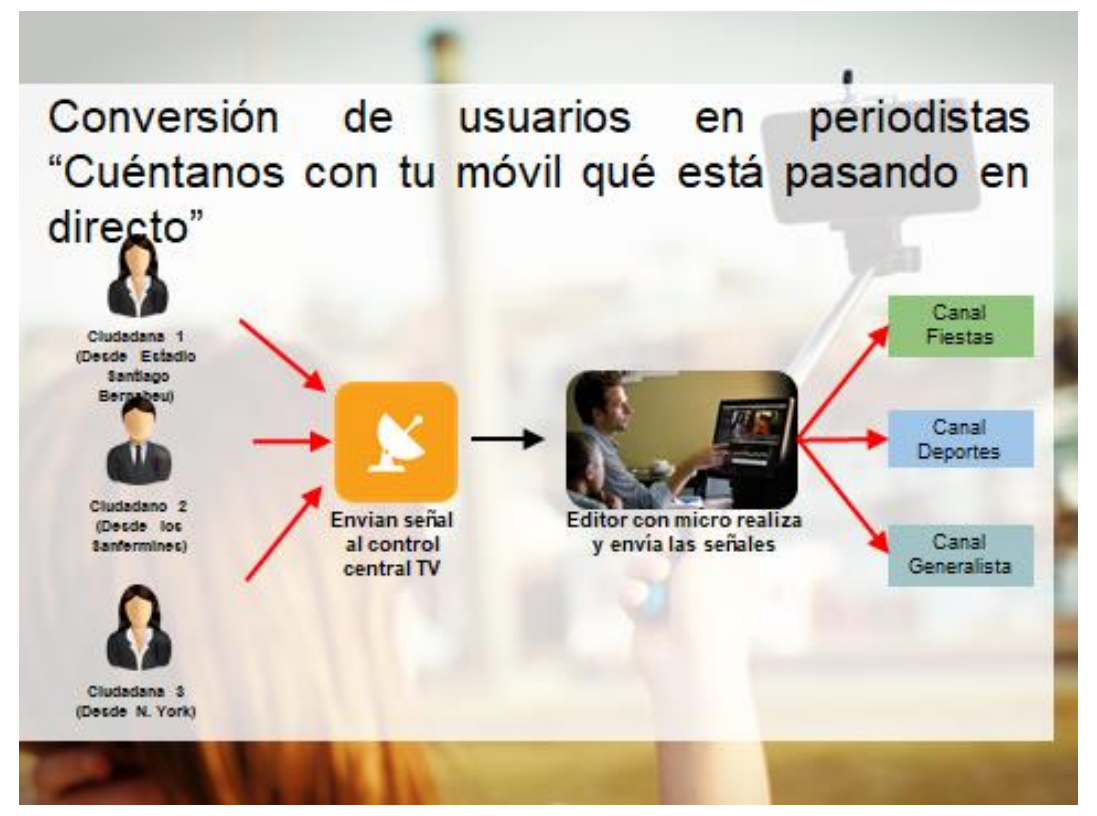

Figura 4. Proceso de envío de señal desde la app hasta el control central de TV donde se reparte la señal a distintos canales

Fuente: elaboración propia

Este protagonismo nace del nuevo papel del espectador, con la ayuda de las herramientas que proceden de la Web 2.0, que está cambiando la forma tradicional de hacer televisión (Mancebo, 2016, p. 330). "Este aumento de la participación evidencia que los ciudadanos han dejado de ser un mero consumidor de contenidos para participar en la construcción y elaboración de los mismos" (Alonso, 2014). El público pasa a ser protagonista de la información también a través de las nuevas tecnologías. Pasa de ser un sujeto pasivo a activo, siempre y cuando, en este caso los editores de televisión consideren oportuno la utilización de esta app con estas funciones.

Esta opción cobra especial relevancia en situaciones extremas, como atentados terroristas, desastres naturales, acontecimientos especiales, etc... pero también para su inclusión en una retransmisión de una fiesta como los Sanfermines, p.ej. La señal de este usuario llegaría a la central y desde allí un editor tiene la opción de incluirla en los informativos o programas, ya sean en directo o para editarla a posteriori.

- Carácter internacional: el streaming es por internet e internet es global. La democratización de internet y el avance actual y futuro de las redes móviles hacen que cualquier persona sea un espectador potencial y las audiencias internacionales superan ampliamente las cotas de los medios tradicionales, limitados a un área geográfica. El streaming une a un país o a una comunidad y puede generar engagement con la cadena de tv a través de un plan estratégico.

\subsection{Reducción de costes}

A la apuesta por la captación de nuevas audiencias, la emisión en streaming une otra característica que la hace única: su menor coste. En el caso concreto de las 
radiotelevisiones, su transformación digital y optimización es crucial para su propio desarrollo en un mercado muy competitivo. En este contexto, hay que producir más barato y mejor para poder competir. El nuevo escenario obliga a los gestores a idear estrategias de ahorro de costes en todas las áreas de la empresa y a una oferta atractiva de servicios para los diferentes públicos que genere audiencias y tráfico digital, lo que determinará un mayor porcentaje de ingresos publicitarios a través de todos sus canales.

A ello se une la evolución tecnológica del streaming, que posibilita una rebaja muy considerable de costes para los grupos mediáticos audiovisuales. La propia idiosincrasia del medio radio, donde los periodistas pueden entrar en directo con un simple teléfono móvil, no ofrece a priori un cambio sustancial en los costes de producción de señal y realización. La radio sigue siendo barata de producir y además la llegada de las últimas plataformas digitales por streaming en directo o contenido bajo demanda permite canales ilimitados de programas para ser consumidos por sus oyentes.

La situación cambia en el caso de las televisiones por el factor señal imagen. Uno de los mayores costes que tienen las televisiones son las retransmisiones en directo, ya sea por la conexión de un periodista en un informativo desde el lugar de los hechos o por el operativo tan grande que hacía falta hasta ahora para poder producir y realizar la señal de un evento. Esa señal por cable terrestre, a través de repetidores o por satélite, llega desde el exterior hasta la sede de la radiotelevisión, y desde allí se distribuye a través de su control central a cada hogar. Ahora esa señal puede llegar a la central de televisión a través de internet vía streaming, y su coste es mucho menor.

El alquiler de una lanzadera de señal (DSNG) y la contratación de la franja satelital tiene un coste mínimo de 1500 euros para la transmisión de un evento de dos horas mientras que el codificador (convertidor de señal de vídeo para internet) y el router (dispositivo que envía esa señal) cuestan 150 euros (emeeme.com). Por tanto, el coste es 10 veces inferior si la trasmisión es por streaming. Los gastos inherentes a la infraestructura necesaria para estas emisiones en directo desde el exterior de los estudios centrales se reducen de forma notable gracias al uso de estas redes IP. Esto conlleva también una rápida implementación del servicio, ya que los plazos de tiempo de puesta en marcha sólo dependen de la producción del contenido.

Otro ahorro viene derivado de la posibilidad del nuevo papel del periodista en los grupos multimedia modernos, ya que el mismo redactor/informador hace una crónica de radio, entra en directo para la televisión y realiza contenido para las RRSS o la web de la cadena. Es el periodista trimedia. Nace con esta nueva tecnología la posibilidad de que un periodista o varios a la vez puedan entrar en directo a través de una app, como en el caso al que nos hemos referido anteriormente de los ciudadanosinformadores. Esos periodistas pueden entrar todos a la vez en un telediario, en un programa informativo o en una retransmisión especial desde distintos lugares del mundo.

La última tecnología permite hoy convertir cualquier dispositivo móvil en una unidad de emisión en directo. Una vez instalada esta app en el móvil de cada periodista y tras 
configurarla en simples pasos se inicia la emisión como si se tratara de la grabación de un vídeo.

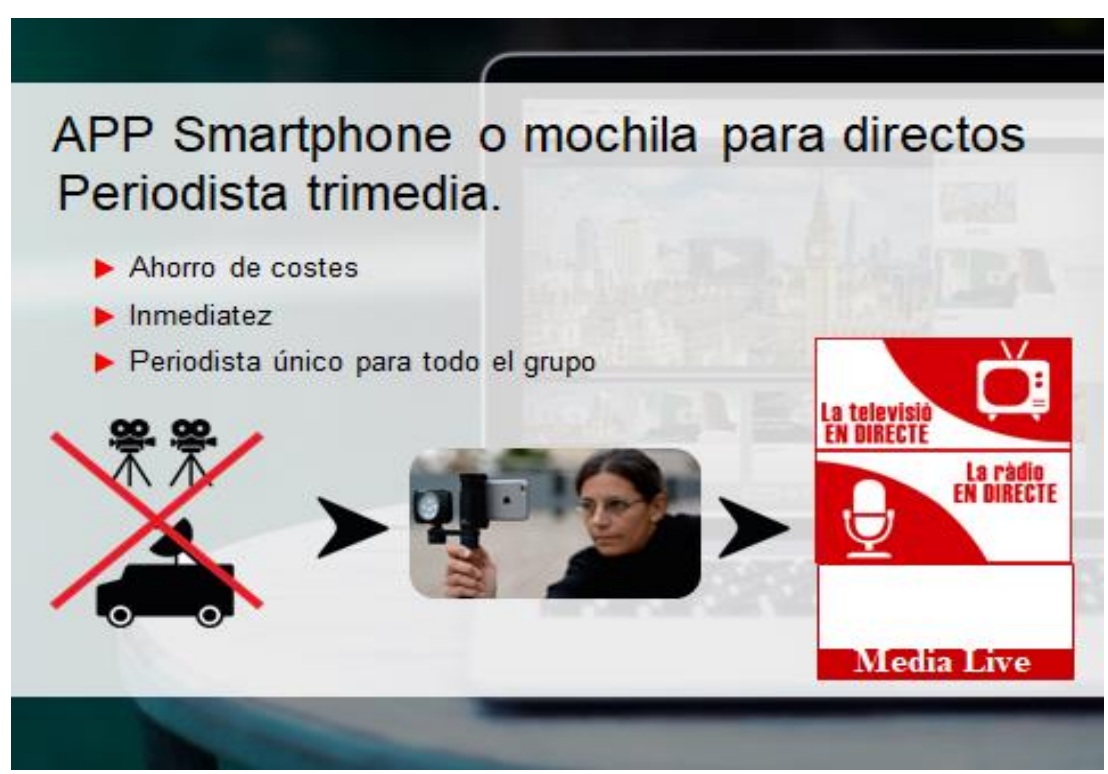

Figura 5. La app sustituye la utilización de lanzaderas de señal

Fuente: elaboración propia

De forma automática, la plataforma digital que la sustenta, recoge el stream de vídeo de cada periodista, genera un player y la publica en la web de cada cadena. Además, el stream de vídeo puede ser capturado por software de broadcasting de terceros, por lo que utiliza dicha emisión como una fuente más de vídeo en una realización profesional. El coste de una app de estas características está en 10.000 euros (shooowit.com). Si tenemos en cuenta los costes de la emisión por satélite en cada ocasión descritos anteriormente, la inversión en esta herramienta también contribuye a reducir el gasto a los grupos multimedia.

A pesar de ello, hay que tener en cuenta que este tipo de distribución digital de contenido audiovisual supone un delay (retraso), un mayor retardo de segundos de recepción de la señal (puede llegar a 10"-15" según la calidad de la señal) cuando la retransmisión es en directo, a diferencia de la retransmisión por satélite, que tiene un desfase de tan apenas 2 o 3 segundos. Ello puede dificultar el diálogo fluido en un informativo en directo entre un presentador y el periodista que está en el lugar de los hechos.

\subsection{Dinamiza el sector audiovisual}

Al menor coste de la emisión en streaming se une el ahorro por la externalización de servicios y su contribución a la dinamización del sector comunicativo. Las empresas de streaming pueden llevar a cabo la producción y realización de la señal televisiva a un coste inferior en un $50 \%$ al de una gran cadena de tv (Óscar Montón, entrevista personal, 15 octubre 2019). Por ejemplo, el precio de un partido de fútbol de $2^{a}$ División A en España se reduce a 4000 euros si externaliza ese servicio (Óscar Montón, entrevista personal, 15 octubre 2019). El operativo incluye: una unidad móvil con seis operadores de cámara, tres auxiliares, un realizador, un técnico de control de cámaras 
(CCU), un técnico de sonido, un operador de sistemas de repeticiones, un operador mezclador, un productor y un jefe de la unidad móvil.

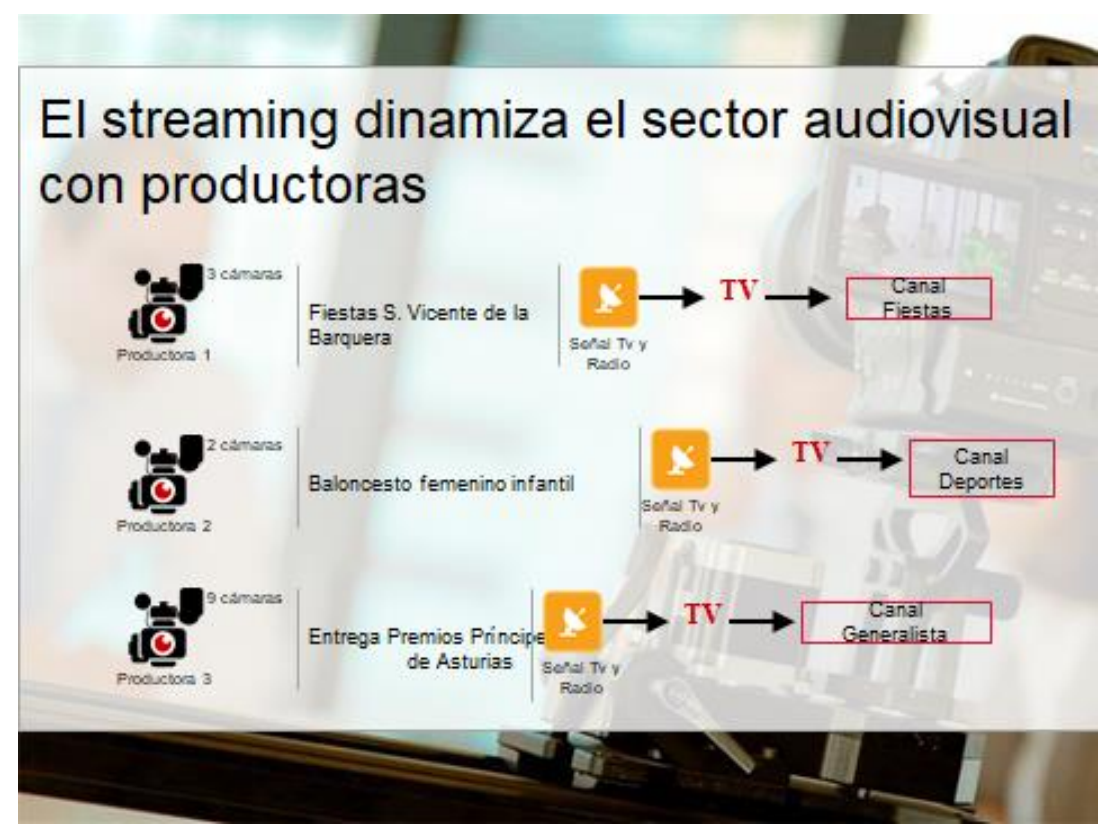

Figura 6. Proceso de envío de señal por streaming hasta el control central de TV donde se reparte la señal a distintos canales.

Fuente: elaboración propia

Si se trata p. ej. de un partido de básquet infantil, el operativo necesario se reduce a la mitad y los costes bajan hasta los 1500 euros. Pero si se tratara de una conferencia o la presentación de un libro, por ejemplo, hay empresas que ofertan ese servicio de 90' con dos cámaras y el editor de realización por 500 euros (www.lasombraproducciones.com). En definitiva, todo ello conlleva nuevas oportunidades en el sector audiovisual y en concreto para las empresas productoras a la hora de poder ofertar sus servicios, que se verían incrementados si cualquier cadena apuesta por la creación de contenidos para su plataforma multicanal bajo la emisión en streaming.

\section{A modo de conclusión}

Las televisiones generalistas en España continúan siendo la primera opción de ocio y por ello tienen un papel relevante en la sociedad. La investigación muestra el crecimiento que ha habido en España y en el resto del mundo del consumo de streaming en los últimos años y refleja su mayor incremento a raíz de la pandemia por la Covid 19. En España ha sido de un 108\% y ello ha supuesto una mayor producción de contenidos mediante streaming tanto por parte de las radiotelevisiones generalistas como de las plataformas de pago, que han visto incrementado sus ingresos por suscripciones.

El trabajo muestra cómo la televisión generalista ha de asumir su nuevo papel como medio de distribución en relación con otras plataformas y a su margen de crecimiento, que está unido a dos factores: su potencialidad para la creación de contenidos propios 
con el uso de la tecnología para streaming y su carácter informativo como mass media, un rasgo diferencial respecto a las plataformas de entretenimiento. Por todo ello tiene un posicionamiento fundamental para formar parte del menú televisivo de los usuarios junto al entretenimiento. El trabajo muestra cómo el nuevo rol de los telespectadores, que quieren interactuar con el emisor, elegir los contenidos y ser protagonistas, ofrece nuevas oportunidades a las televisiones generalistas para interactuar con sus públicos.

La investigación supone un avance del conocimiento en esta materia porque pone de manifiesto cómo las nuevas herramientas tecnológicas constituyen nuevas oportunidades para la televisión, ya que hay apps que permiten al periodista y al telespectador entrar en directo en un informativo o un programa a través de su teléfono móvil. De esta forma, la tecnología a través del streaming cumple otra demanda de la sociedad. El público pasa a ser protagonista de la información, no un mero consumidor de contenidos. Hay un aumento de la participación de los ciudadanos, que quieren ser activos, elaborar contenidos y ello contribuye a una mayor democratización del streaming.

Las televisiones generalistas disponen de alta tecnología para emitir por streaming a través de su web con canales ilimitados a través de multiplataformas digitales para llegar a todos sus públicos y generar tráfico con posibilidades enormes de variedad de contenidos. La emisión por streaming, por Internet, es además 10 veces inferior a la trasmisión satelital, lo que abarata costes de producción de señal y de contenidos, y dinamiza el sector audiovisual, ya que las productoras de televisión pueden ofrecer esos servicios a las televisiones generalistas.

Los resultados también confirman que 9 de cada 10 internautas en España suelen conectarse a Internet a través de su teléfono móvil de manera habitual y el formato que más se consume son los vídeos cortos y videos bajo demanda, lo que da más posibilidades de negocio a las televisiones generalistas creadoras de contenido.

Para futuras investigaciones cabría analizar si estas perspectivas de futuro y los retos que tiene ante sí son aprovechados por las cadenas de televisión. Otros trabajos pueden ir encaminados a saber cómo las cadenas de televisión monetizan la inversión de los nuevos contenidos y qué políticas de e-commerce utilizan estas empresas, estudiando si hay distinción entre las políticas comerciales que utilizan las cadenas privadas y las públicas.

\section{Bibliografía}

AIMC (Asociación para la Investigación de Medios de Comunicación, 2020) https://www.aimc.es/a1mc-c0nt3nt/uploads/2020/01/marco2020.pdf

Alonso González, M. (2014) Audiencia Social: el telespectador comienza a participar en los contenidos televisivos. Ámbitos: Revista internacional de comunicación, 25, 1-16.

https://idus.us.es/xmlui/handle/11441/66881

http://hdl.handle.net/11441/66881 
Antolín-Prieto, R. (2012). Youtube como paradigma del vídeo y la televisión en la web 2.0 [Universidad Complutense Madrid]. https://eprints.ucm.es/16111/1/T33817.pdf

Bergillos, I. (2015). Participación de la audiencia y televisión en la era digital. Propuesta de análisis y evolución de las invitaciones a la participación en la tdt y en otras plataformas. [Universidad Autónoma de Barcelona] https://ddd.uab.cat/pub/tesis/2015/hdl 10803 308326/ibg1de.pdf

Caldwell, J. (2006). Critical Industrial Practice: Branding, Repurposing, and the Migrattory Patterns of Industrial Texts. Television \& New Media, 7(2), 99-134. https://journals.sagepub.com/doi/10.1177/1527476403255811

Campos, F. (2017). El valor social de la televisión abierta. En: Gutiérrez, Eladio (coord.). Televisión abierta. Situación actual y tendencias de futuro de la TDT. Colegio Oficial de Ingenieros de Telecomunicación. https://www.coit.es/sites/default/files/informes/pdf/libro blanco sobre la funcion social de la tdt final 50aniversario.pdf

Comisión Nacional de los Mercados y la Competencia (CNMC). Panel de Hogares (2019, último cuatrimestre) https://www.cnmc.es/

Creeber, G., Hills, M. (2007). Tvlll: Into, or Towards, a New Television Age? New Review of Film and Television Studies, 5(1), 1-4. https://doi.org/10.1080/17400300601140118

Curtis, R. C. (2008, junio 5). Internet romperá los límites en la televisión de alta definición [El

país.com]. http://www.elpais.com/articulo/elpciblse/20080605elpciblse 6/Tes

Entrevista de Ignazio Zafra. Citada en Tesis Doctoral. La comunicación a través del video streaming: análisis del caso YouTube. https://hera.ugr.es/tesisugr/19587703.pdf

Diego-González, P., Guerrero-Pérez, Enrique y Etayo-Pérez, Cristina (2014). Televisión conectada en España: contenidos, pantallas y hábitos de visionado. Revista Mediterránea de Comunicación, 5(1), 179-199. https://doi.org/10.14198/MEDCOM2014.5.1.10

Empresa digital Guide lonos. https://www.ionos.es/

Empresa productora de streaming. www.lasombraproducciones.com

Empresa streaming. https://www.timetoast.com/

Empresa tecnológica https://conceptoweb-studio.com/

Empresa tecnológica Shooowit. www.shooowit.com 
Estudio "DIMENSION 2020: Media \& Me" https://kantar.com/es/inspiracion/publicidad-y-medios/la-guerra-del-streaming/

Ferrando Casanova, J. L. O. (2019, octubre 29). CEO Shoowit [Entrevista personal].

Fundéu BBVA. https://www.fundeu.es/recomendacion/over-the-top/

Gallardo-Camacho, J., Lavín, E., Fernández-García, P. (2016) Los programas de televisión deportivos y su relación con la audiencia social en Twitter en España. Revista Latina de Comunicación Social, 71, 272-286. http://www.revistalatinacs.org/071/paper/1095/15es.html

González, Í. (2020) Aumenta un 108\% el consumo de streaming en España. Artículo publicado en Legameb el 3 de diciembre de 2020. https://legameb.25gramos.com/aumenta-un-108-el-consumo-de-streaming-enespanal

Guerrero Pérez, Enrique. (2018) La fuga de los millennials de la televisión lineal. Revista Latina de Comunicación Social, 73, 1231- 1246. http://www.revistalatinacs.org/073paper/1304/63es.html https://doi.org/10.4185/RLCS-2018-1304

Hootsuite \& we are social (Report 2020) https://hootsuite.com/pages/digital-in-2020

Informe Anual del Sector de los Contenidos Audiovisuales en España de 2019 https://www.ontsi.red.es/sites/ontsi/files/2020-01/PresentacionContenidos Digitales2019 0.pdf

Informe Digital $\quad$ Tv $\quad$ Research 2020 https://www.digitaltvresearch.com/ugc/Global\%20AVOD\%20Forecasts $\% 202020 \%$ 20TOC toc 288.pdf

Izquierdo, J. (2017) ¿Quién ve la televisión? Revisión del modelo generalista en el espacio mediático convergente. Trípodos. 40, 31 - 33 . Barcelona. http://www.tripodos.com/index.php/Facultat Comunicacio Blanquerna/article/view $\underline{1448}$

Kuschevatzky, A. Referencia a este productor de cine en el artículo "El tsunami del consumo on line para piratas y autores", de Natali Schejtman publicado en "La Nación" (5 de noviembre 2017) http://www.lanacion.com.ar/2078770-el-tsunamidel-consumo-online-un-desafio-para-piratas-y-autores

López, M. (2010) Google quiere entrar en nuestros televisores. https://www.genbeta.com/multimedia/google-quiere-entrar-en-nuestros$\underline{\text { televisores }}$

Mancebo García, M. (2016) Innovación en formatos audiovisuales. El uso de herramientas de live streaming en Antena 3 y Univision. Miguel Hernández 
Communication Journal. 325-349. http://mhjournal.org/\%5blink\%20del\%20artículo\%5d

Manual streaming NPLA. Pool de Noticias de América Latina. p.7 https://www.npla.de/files/Manual Streaming NPLA-2010 ES.pdf www.npla.de

Marín Amatller, A. (2005). Vídeo digital en Internet. Barcelona.

Medina Salgado, C. (2016) Los millennials, su forma de vida y el streaming. Revista Gestión y Estrategia. 50, 126. https://core.ac.uk/download/pdf/83079880.pdf

Meiker, G. y Young, S. (2008) Beyond Broadcasting? TV for the Twenty-First Century. Media International Australia. 67-70. https://doi.org/10.1177/1329878X0812600108

Montón Hueso, Ó. CEO Emeeme Videoproducciones. (Entrevista personal, 15 de octubre 2019).

Productora de televisión Ememe Videoproducciones. www.emeeme.com

Quintas Froufe, N. \& González Neira, A. (2014) Audiencias activas: participación de la audiencia social en la televisión. Comunicar, 43, 83-90. http://goo.gl/h3M8Um

Roams (2020) https://www.hobbyconsolas.com/noticias/consumo-streaming-espanaaumenta-108-ciento-767461 